\title{
The presence of CLL-associated stereotypic $B$ cell receptors in the normal $B C R$ repertoire from healthy individuals increases with age
}

Alice F. Muggen ${ }^{1}$, Madelon de Jong ${ }^{1}$, Ingrid L. M. Wolvers-Tettero ${ }^{1}$, Martine J. Kallemeijn ${ }^{1}$, Cristina Teodósio ${ }^{1,2}$, Nikos Darzentas ${ }^{3,4}$, Ralph Stadhouders ${ }^{5}$, Hanna IJspeert ${ }^{1}$, Mirjam van der Burg ${ }^{1,6}$, Wilfred FJ van IJcken ${ }^{7}$, Jan A. N. Verhaar ${ }^{8}$, Wayel H. Abdulahad ${ }^{9}$, Elisabeth Brouwer ${ }^{9}$, Annemieke M. H. Boots ${ }^{9}$, Rudi W. Hendriks ${ }^{5}$, Jacques J. M. van Dongen ${ }^{1,2}$ and Anton W. Langerak ${ }^{1 *}$

\begin{abstract}
Background: Aging is known to induce immunosenescence, resulting in alterations in both the innate and adaptive immune system. Here we evaluated the effects of aging on B cell subsets in peripheral blood of 155 immunologically healthy individuals in four age categories (range 20-95y) via multi-parameter flow cytometry. Furthermore, we studied the naive and antigen-experienced $B$ cell receptor $(B C R)$ repertoire of different age groups and compared it to the clonal BCR repertoire of chronic lymphocytic leukemia (CLL), a disease typically presenting in elderly individuals.

Results: Total numbers and relative frequencies of $B$ cells were found to decline upon aging, with reductions in transitional B cells, memory cell types, and plasma blasts in the $70+y$ group. The BCR repertoire of naive mature B cells and antigen-experienced B cells did not clearly alter until age 70y. Clear changes in IGHV gene usage were observed in naive mature $B$ cells of $70+y$ individuals, with a transitional pattern in the 50-70y group. IGHV gene usage of naive mature $B$ cells of the 50-70y, but not the $70+y$, age group resembled that of both younger (50-70y) and older $(70+y)$ CLL patients. Additionally, CLL-associated stereotypic BCR were found as part of the healthy control BCR repertoire, with an age-associated increase in frequency of several stereotypic BCR (particularly subsets \#2 and \#5).

Conclusion: Composition of the peripheral B cell compartment changes with ageing, with clear reductions in nonswitched and CD27 + lgG + switched memory B cells and plasma blasts in especially the $70+y$ group. The BCR repertoire is relatively stable until 70y, whereafter differences in IGHV gene usage are seen. Upon ageing, an increasing trend in the occurrence of particular CLL-associated stereotypic BCR is observed.
\end{abstract}

Keywords: Aging, B-lymphocyte, BCR repertoire, CLL, Stereotypic BCR

\section{Background}

Changes in the immune system related to aging generally lead to increased susceptibility to infections, poor responses to new and evolving pathogens, poor vaccination responses, and higher incidence of autoimmune disorders and malignancies [1, 2]. This decline in function of the immune system, also referred to as immunosenescence, is the result

\footnotetext{
*Correspondence: a.langerak@erasmusmc.nl

'Department Immunology, Laboratory Medical Immunology, Erasmus MC, Dr. Molewaterplein 40, 3015, GD, Rotterdam, The Netherlands

Full list of author information is available at the end of the article
}

of alterations occurring in both innate and adaptive immunity [3].

Age-related changes in humoral immune responses have generally been ascribed to defects in the $\mathrm{T}$ cell compartment and a lack of $\mathrm{T}$ cell help for $\mathrm{B}$ cell function [3]. Nevertheless, mouse studies do provide evidence for changes in the $\mathrm{B}$ cell compartment itself during aging. Although total B cell numbers did not alter much, shifts in the distribution of functional subsets were apparent with old age. In fact, in old mice nearly $100 \%$ of splenic B cells exhibited an antigen-experienced phenotype [4]

(c) The Author(s). 2019 Open Access This article is distributed under the terms of the Creative Commons Attribution 4.0 International License (http://creativecommons.org/licenses/by/4.0/), which permits unrestricted use, distribution, and 
and circulating immunoglobulins (Ig) were predominantly derived from post-germinal center B cells, as deduced from the presence of somatic hyper mutations (SHM) [5].

In human, age-related alterations in peripheral blood (PB) B cell subset distribution have also been reported, with circulating CD19+ B cells declining in absolute numbers and frequencies [6-10]. In some studies numbers and percentages of $\mathrm{CD} 27+$ memory $\mathrm{B}$ cells were found to decline $[7,8]$, whereas others showed an increase of these cells [10-12]. Similarly, numbers and percentages of naive CD27-IgD+ B cells were found to decrease by some studies $[9,10,12]$, whereas others reported an increase $[7,8]$. These inconsistent results may be explained by different $\mathrm{B}$ cell subset definitions and/or by large inter-individual variations in the studied age groups $[11,13]$.

Changes in B cell subsets during aging will likely also impact on B cell receptor (BCR) repertoire diversity. Indeed, in several mouse models age-related changes in the naive BCR repertoire were reported [14]. In some elderly humans, Ig heavy chain (IGH) complementarity determining region 3 (HCDR3) spectratyping analysis of PB B cells revealed a significant loss of diversity, which was associated with poor health status and poor survival [13]. Conflicting data, however, were reported on SHM in IGHV genes of the memory $\mathrm{B}$ cell compartment upon aging, varying from increased mutation rates in IgG+ but not in IgM+ PB memory $\mathrm{B}$ cells, to increased mutation rates in IgM+ memory B cells but not in other tonsillar subsets $[15,16]$.

Introduction of next generation sequencing (NGS) technologies has opened new possibilities to analyze the aging BCR repertoire, particularly in the light of immune diseases that typically arise in elderly. One category of immune diseases with a higher change to develop in elderly humans are B cell malignancies, with chronic lymphocytic leukemia (CLL) being the most common type. Notably, in about one-third of CLL patients, quasiidentical (stereotypic) BCRs are observed, which are characterized by restricted IGHV, IGHD, and IGHJ gene usage plus similarities in HCDR3 length and amino acid sequence [17]. One study reported on stereotypic BCRs within the normal IGHV1-69-IGHJ6 repertoire [18]. Litthe is known however about the overall existence of CLL-associated stereotypic BCRs in the normal BCR repertoire of different age groups. We hypothesize that these CLL-associated stereotypic BCR could be a reflection of changes in $\mathrm{B}$ cell subset distribution and the normal $B C R$ repertoire upon aging.

In this study we determined absolute numbers and relative distribution of PB B cell subsets in healthy individuals of different age categories. Additionally, we used NGS to investigate the BCR repertoire of naive mature $B$ cells and different types of antigen-experienced B cells in healthy individuals upon aging. Finally, we compared IGHV gene usage of the normal BCR repertoire of different age groups with that of CLL leukemic cells and evaluated the occurrence of CLL-associated stereotypic BCR in the aging normal $\mathrm{BCR}$ repertoire.

\section{Results \\ Alterations in peripheral blood B cell subpopulations are minor upon aging}

To study B cell dynamics during aging, we performed extensive flow cytometric immunophenotyping of peripheral blood (PB) cells in a cohort of 155 immunologically healthy individuals of $20-95$ years $[<50 \quad(n=47)$, 50-60 $(n=31), 60-70(n=45)$, and $70+(n=32)]$.

To validate our cohort, we first evaluated age-related dynamics of the total white blood cells and lymphocyte subpopulations (Fig. 1; Additional file 1: Figure 1). White blood counts (WBC) remained stable across the age groups (Fig. 1A). Although differences were not significant, there was a trend that the absolute numbers of lymphocytes was lower in the 50-60y and 60-70y groups than in the $<50 y$ group (Additional file 1: Figure S1). Both absolute and relative numbers of naive CD8+ T cells significantly declined $>50 \mathrm{y}$ of age, whereas absolute and relative numbers of CD8+ effector (TemRA) T cells clearly increased with increasing age and CD8+ effector memory (TemRO) $\mathrm{T}$ cells remained stable (data not shown). CD4+ T cell and NK cell numbers did not alter between the age groups (Additional file 1: Figure S1). These data are in line with previously reported data on $\mathrm{T}$ and NK cells [19], thus supporting the validity of our cohort for evaluating B cell aging effects.

Next, we focused on the composition of the B cell compartment (see Methods, Additional file 6: Table S1, Additional file 2: Figure S2) in the different age groups. The total B cell numbers and relative frequencies of B cells (as fraction of total lymphocytes) slightly declined during aging, resulting in a significant difference between the $<50 \mathrm{y}$ and $70+y$ groups (Fig. 1B-C). More specifically, we observed a significant reduction in absolute numbers of transitional B cells in the $70+y$ group, as well as reductions in the non-switched and CD27 + IgG+ switched memory B cell populations, and plasma blasts in the two oldest age categories (Fig. 1D-M). For naive mature B cells and all other types of memory B cells no clear alterations in absolute numbers were noted upon aging (Fig. 1D-M). The overall PB B cell subset distribution displayed only minor shifts between different age groups (Fig. 1N), which was mainly reflected by the significantly lower frequencies of non-switched memory B cells and plasma blasts upon increasing age (Additional file 3: Figure S3).

We then looked into CD $5+\mathrm{CD} 43+$ chronically activated $B$ cells, as these have been associated with CLL and CLL-like MBL that typically appear in elderly [2022]. We noted a small but significant increase in relative 


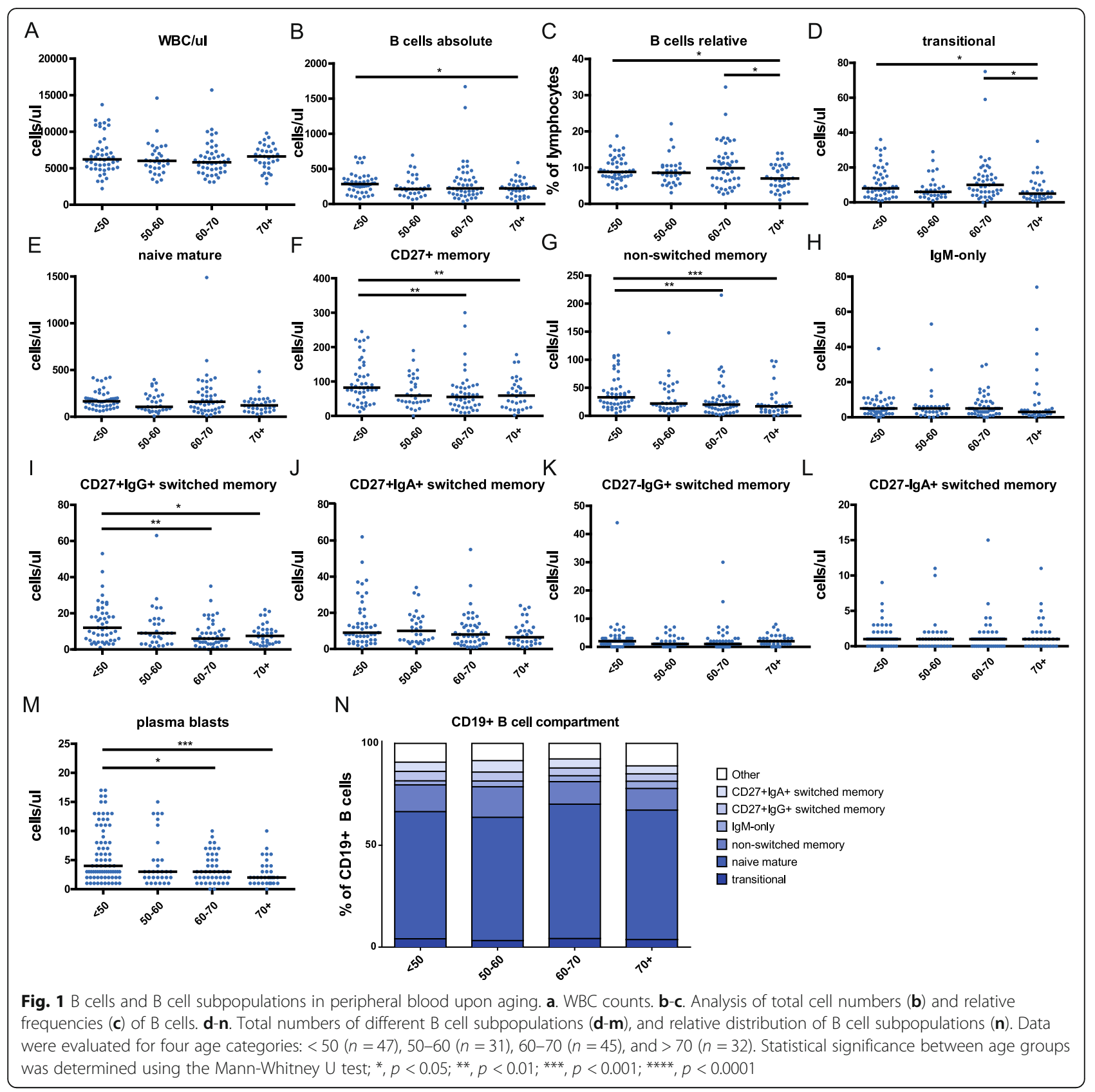

frequencies of CD5 + CD43+ B cells in the $70+y$ group (when compared with the 60-70y age group), together with a trend towards increased absolute numbers of these cells (Fig. 2A-B). Next, we also evaluated CD21 ${ }^{\text {low }}$ B cells, as high numbers of these cells have been associated with autoimmune disease [23]. Notably, a significant increase of $\mathrm{CD} 21^{\text {low }}$ cell numbers and relative frequencies was seen between the 50-60y and 60-70y groups, which normalized again in the $70+y$ group (Figs. 2C-D).

Taken together, our B cell subpopulation analysis mostly showed a decline in transitional B cells, non-switched and CD27 + IgG+ switched memory B cells, and plasma blasts in elderly. The frequency of $\mathrm{CD} 21^{\text {low }} \mathrm{B}$ cells appeared to be increased in 60-70y group.

Composition of the $B C R$ repertoire of naïve mature $B$ cells is stable until 70y but shows changes in the $70+y$ group Our next aim was to see which differences occur in the $\mathrm{BCR}$ repertoire of healthy donors during aging. To this end, we first sorted antigen-inexperienced naive mature B cells of healthy controls in the $<50 y, 50-70 y$, and $70+$ y groups ( $n=4-5$ per age group) and analyzed unique IGHV-IGHD-IGHJ sequences and their HCDR3 regions (Additional file 6: Table S2). Despite slight variations in 


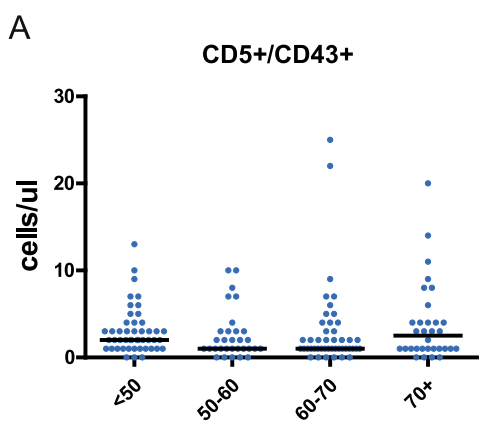

B
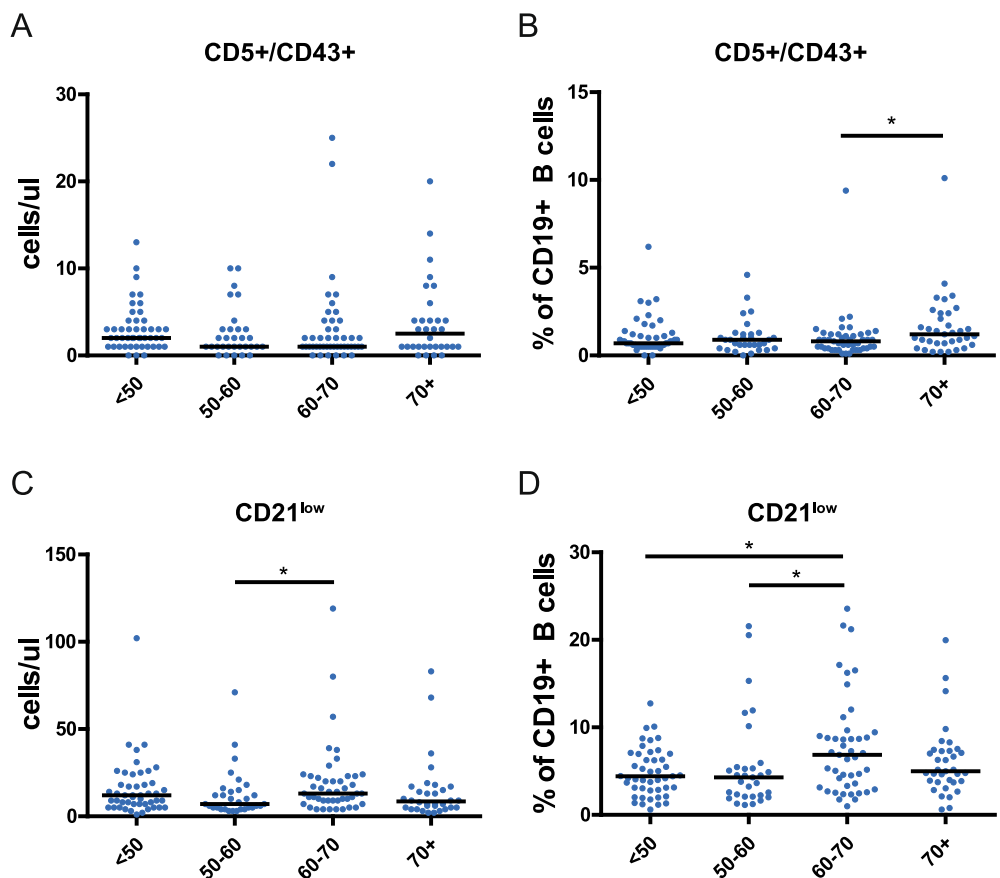

D

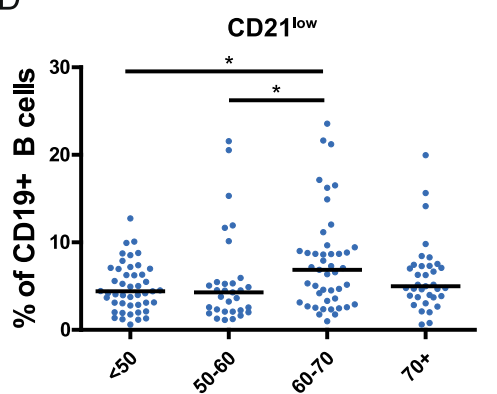

Fig. $2 \mathrm{CD} 43+\mathrm{CD} 5+\mathrm{B}$ cells and CD21 ${ }^{\text {low }} \mathrm{B}$ cells in peripheral blood upon aging. Analysis of total cell numbers $(\mathbf{a}, \mathbf{c})$ and relative frequencies (b,d) of CD5 + CD43+ B cells and CD21 low B cells, respectively. Data were evaluated for four age categories: $<50(n=47), 50-60(n=31), 60-70(n=45)$, and $>70$ $(n=32)$. Statistical significance between age groups was determined using the Mann-Whitney $U$ test;,$p<0.05 ;{ }^{* *}, p<0.01 ;{ }^{* * *}, p<0.001 ;{ }^{* * *}, p<0.0001$

the mean HCDR3 lengths between the $<50 y$ (53.0 nucleotides), 50-70y (54.5 nucleotides), and $70+y$ (46.1 nucleotides) groups, the overall HCDR3 profiles showed no significant differences (Fig. 3A).

When evaluating gene usage, differences in IGHV subgroup usage between the $<50 \mathrm{y}$ and $50-70 \mathrm{y}$ groups appeared limited, but we did find a marked increase in IGHV1 and IGHV5 subgroup usage and a decrease in IGHV4 subgroup usage in the $70+y$ donors (Fig. 3B). Upon further examination of IGHV gene usage, significant differences were mostly noted in the $70+y$ age group, with IGHV1-18, IGHV1-46, IGHV1-69, and IGHV5-51 gene usage being significantly higher, and IGHV4-34, IGHV4-39, and IGHV4-59 usage being significantly lower (Fig. 3C), which could not be explained by small clonal proliferations. That said, the most commonly used IGHV gene in all three age groups appeared to be the IGHV3-23 gene, followed by IGHV3-21 (Fig. 3B). We did not detect clear differences in IGHD and IGHJ gene usage between any of the three age groups.

Collectively, our data from healthy controls of different age groups suggest that the $\mathrm{BCR}$ repertoire of naive mature B cells is relatively stable until $70 y$. In contrast, in the $70+y$ group IGHV gene usage does differ, which most probably should be interpreted as an aging effect, since there were no other indications that can explain this difference.
Differences in BCR repertoire of memory B cell subpopulations are minor between $<50 y$ and 50-70y age groups

To evaluate age effects in the antigen-selected BCR repertoire, we then focused on non-switched, IgM-only, and CD27 + IgG+ switched memory B cells, which are all antigen-experienced cells though arising via distinct activation routes. Some of the memory B cell populations are so small that they can only be sorted from buffy coats of healthy donors ( $n=4-5$ per age group); unfortunately blood donors are only allowed to give blood until $70 y$, so we could not the study the $70+y$ age group. Mean HCDR3 lengths did not differ significantly between the $<50 \mathrm{y}$ and 50-70y age groups (Additional file 4: Figure S4). IGHV, IGHD, and IGHJ gene usage and combined IGHV / IGHJ patterns of nonswitched, IgM-only, and CD27 + IgG+ memory B cells did not show significant differences either between these two age groups (Additional file 5: Figure S5). Notably, when analyzing SHM frequencies for these memory B cell subpopulations, we did detect a higher mutation rate for non-switched and IgM-only memory B cells in the $50-70 y$ group, whilst a small reduction in mutation frequency was seen in CD27 + IgG+ switched memory B cells (Additional file 5: Figure S5).

Collectively these data show that the BCR characteristics of memory B cell subpopulations do not differ statistically between the $<50 \mathrm{y}$ and $50-70 \mathrm{y}$ groups. 
naive mature

A

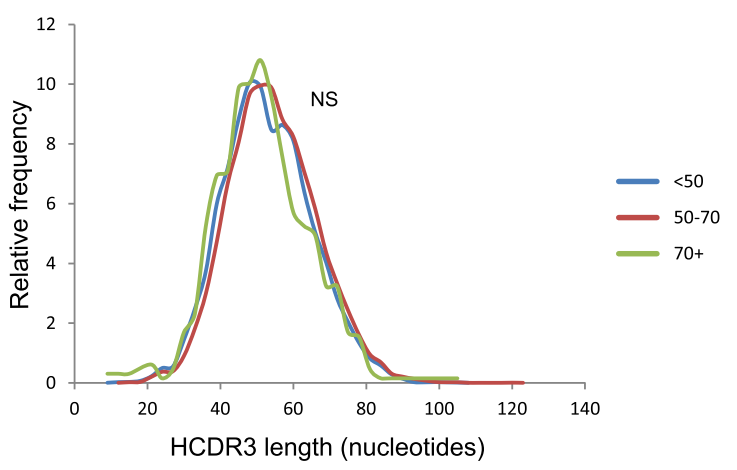

B $<50$
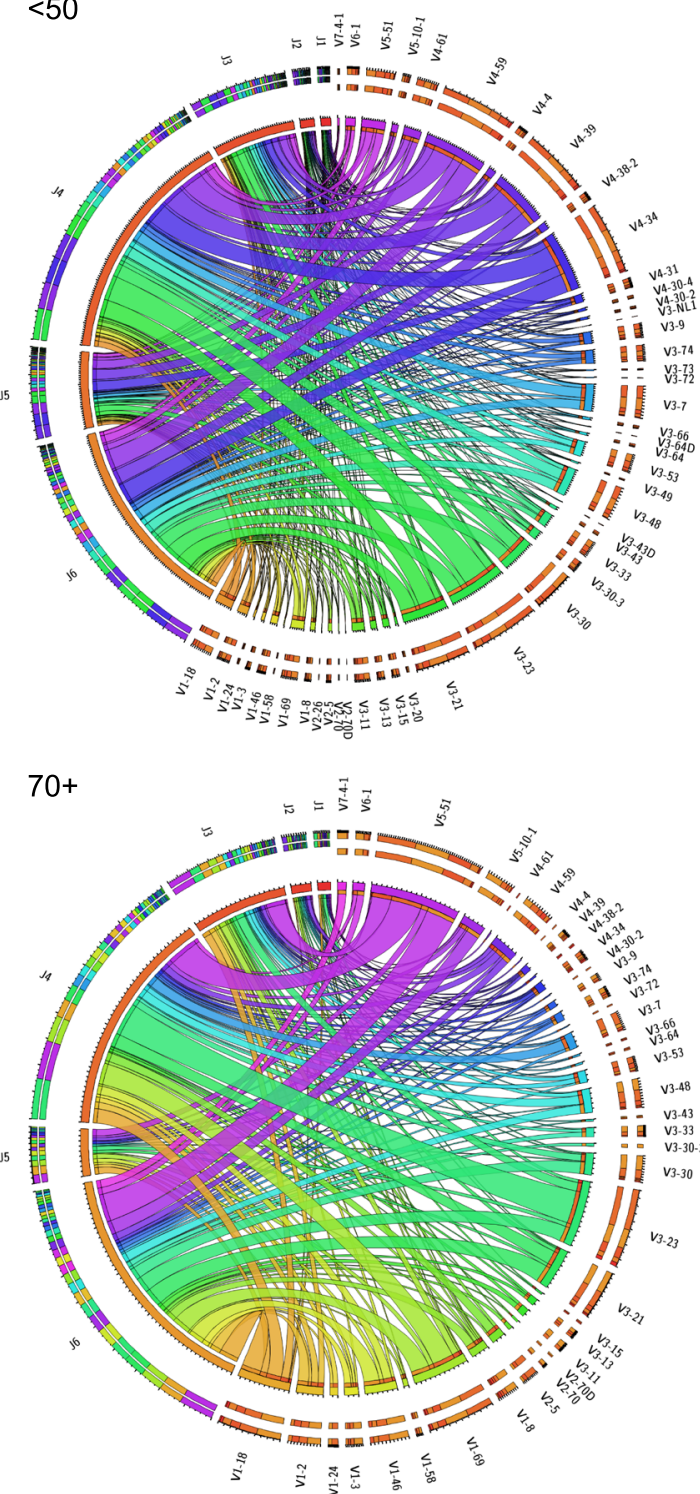

$50-70$

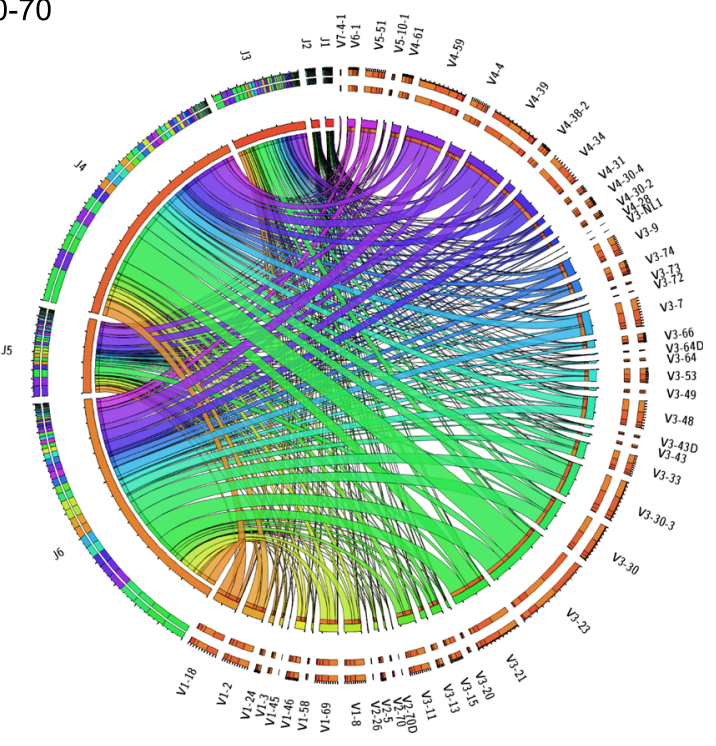

C

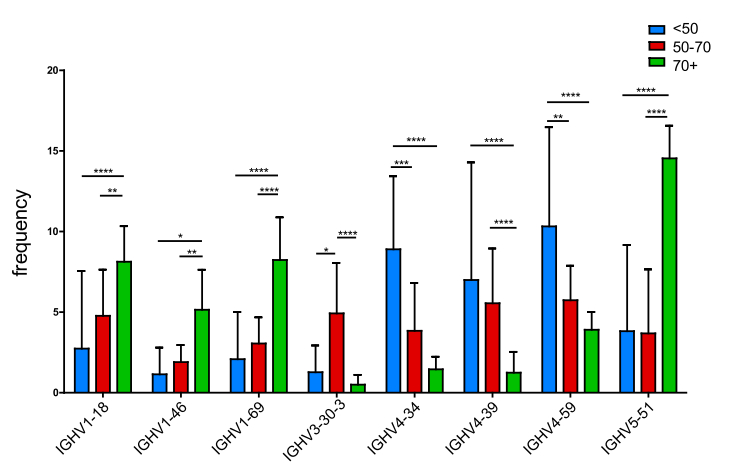

Fig. 3 (See legend on next page.) 
(See figure on previous page.)

Fig. 3 BCR repertoire analysis in naive mature B cells upon aging. a. Comparison of HCDR3 lengths in $<50,50-70$, and $70+y$ age categories. Statistical significance was determined using the Kolmogorov-Smirnov test. $\mathbf{b}$. Circos plots of the combination of IGHV-IGHJ gene usage in <50, 50-70, and $70+y$ age categories. c. Differences in IGHV gene usage frequencies upon aging. Statistical significance between different age groups was determined using the two-way ANOVA test (with Bonferroni multiple comparisons correction); ${ }^{*}, p<0.05 ;{ }^{* *}, p<0.01 ;{ }^{* * *}, p<0.001$; ****, $p<0.0001$

IGHV gene usage in the clonal BCR repertoire of CLL patients is largely comparable to IGHV genes in naive mature $B$ cells of 50-70y individuals

As the BCR is known to play an important role in disease onset and prognosis of CLL, which normally develops at elderly age (average $70+y$ ), we then asked whether overall IGHV gene usage in CLL patients of different age groups would reflect the BCR repertoire of normal B cells of the same age groups. To this end we evaluated IGHV gene usage in different B cell subsets of healthy controls of different age groups and compared the profiles with Sanger sequencing-based data of a cohort of 920 CLL patients (Fig. 4).

Overall IGHV gene usage profiles did not differ significantly between different types of normal B cell subpopulations, such as naive mature B cells, nonswitched memory B cells, and IgM-only memory B cells, in the 50-70y age group ( $p=0.99-1$ in all comparisons). Unfortunately, no such comparison was possible in $70+y$ individuals, due to the lack of available data from non-switched and IgM-only memory B cells for this age group. However, overall IGHV gene usage profiles in naive mature $B$ cells of healthy individuals did clearly vary between the $50-70 \mathrm{y}$ and $70+\mathrm{y}$ groups $(p=0.005)$.

When we next compared overall IGHV gene usage profiles between CLL patients of the 50-70y and the $70+y$ groups we did not observe a significant difference $(p=0.995)$. The IGHV profiles of naive mature $B$ cells and CLLs in the 50-70y group appeared to look rather similar $(p=0.574)$, albeit with a more dominant IGHV1-69 and IGHV4-34 usage in CLL. In contrast, naive mature $\mathrm{B}$ cell and CLL IGHV gene usage profiles in the $70+y$ groups were clearly different $(p<0.0001)$, while the overall IGHV profile in $70+y$ CLL was in fact rather similar to that of naive mature B cells of the 50-70y age group $(p=0.110)$. Furthermore, overall IGHV profiles in the $50-70 \mathrm{y}$ group appeared clearly different between CLL and non-switched memory B cells $(p=0.028)$ or IgM-only memory B cells $(p=0.004)$.

These data indicate that the overall IGHV gene usage profile in CLL patients, irrespective of the age of presentation, is similar to naive mature B cells of especially the $50-70 y$ control group. The overall IGHV profile of the $70+y$ control group is different without obvious explanation as mentioned above.

\section{CLL-associated stereotypic BCR are present in naive mature $B$ cells and increase with age}

In view of the occurrence of quasi-identical (stereotypic) BCR with similar IGHV / IGHD / IGHJ and HCDR3 features in CLL clones of different patients, we then investigated whether we could also detect stereotypic BCR in healthy individuals of different age groups. To identify CLL-related stereotypic BCRs in the normal repertoire of healthy controls of different age groups, a reference database of stereotypic BCR from CLL patients [24] was used for assigning individual IGH sequences from healthy controls. With this algorithm we could indeed identify CLLassociated stereotypic BCRs based on HCDR3 characteristics in the repertoire of naive mature $\mathrm{B}$ cells, and also of non-switched, IgM-only, and CD27 + IgG+ switched memory B cells (Fig. 5). The presence of stereotypic BCR receptors in naive mature $B$ cells showed an increasing trend with age and was most apparent in the $70+y$ group (Fig. 5A). In naive mature $B$ cells, the most prominent stereotypic BCR belonged to CLL subsets \#2, \#5, and \#64B (Fig. 5B). In non-switched memory B cells the most frequently found stereotypic BCRs concerned CLL subsets \#2 and \#14 (Fig. 5C). The CLL\#14 BCR also appeared most prominent in IgM-only and CD27 + IgG + switched memory B cells, especially in the 50-70y category (Fig. 5D-E).

From these findings we conclude that CLL-associated stereotypic BCRs are present in the normal BCR repertoire during life and that their frequency showed an increasing trend upon aging.

\section{Discussion}

Here we evaluated age-related changes in B cell subset composition as well as BCR repertoire with a focus on CLL-associated stereotypic BCR usage. In the most elderly $(70+y)$ individuals we observed a decline in absolute numbers of transitional $\mathrm{B}$ cells, total memory $\mathrm{B}$ cells, and plasma blasts. The BCR repertoire of naive mature B cells and distinct memory B cell populations was found to be relatively stable until $70 y$. In naive mature B cells of the $70+y$ group differences were noted, especially with respect to IGHV gene usage. Notably, IGHV gene usage in the clonal BCR repertoire in CLL did not differ with the age of presentation of the CLL patients, and largely resembled naive mature B cells of the $50-70 y$ group rather than the $70+y$ group. Finally, CLL-associated stereotypic BCR were found as part of 


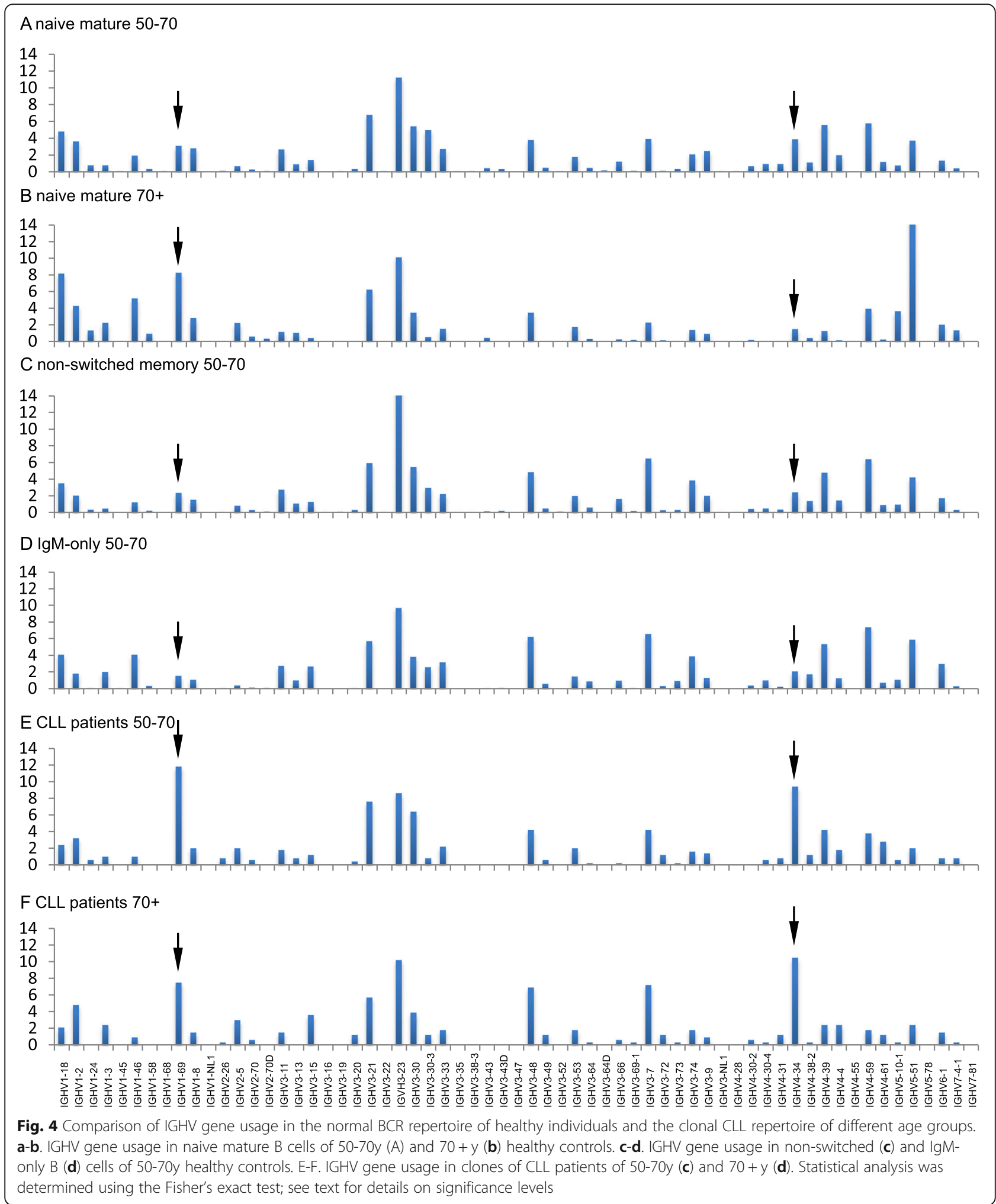

the BCR repertoire of healthy individuals and their frequencies increased with age.

The observed decline in total B cell numbers in the $70+$ $y$ group could result from a reduced output from the bone marrow $[25,26]$. An alternative explanation could be the reduction in $\mathrm{CD} 27+$ antigen-experienced $\mathrm{B}$ cell subpopulations. Non-switched and CD27 + IgG+ memory B cells together make up the majority of the $\mathrm{CD} 27+$ memory $\mathrm{B}$ cell 


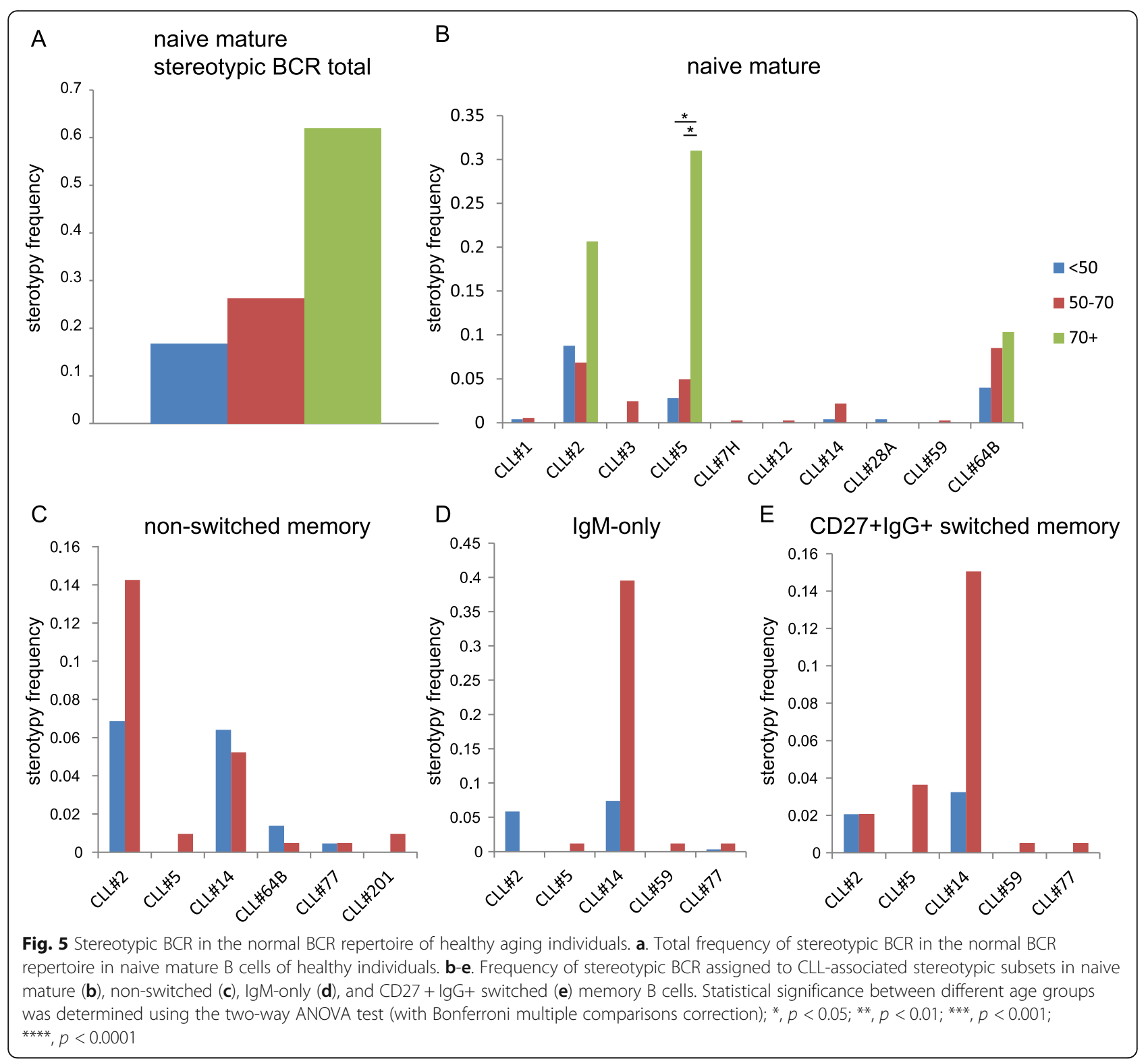

compartment, and were previously described to decline upon aging [6]. The decline in non-switched memory B cells could underlie the higher vulnerability to diseases caused by encapsulated bacteria like Streptococcus pneumoniae in elderly [27]. CD27 + IgG+ memory B cells are mainly, albeit not exclusively, formed in $\mathrm{T}$ cell-dependent immune responses and play a role in recall responses to previously encountered pathogens [28]. The reduction of plasma blasts upon aging is in line with earlier observations [29] and fits the lower immunoglobulin levels in the circulation as reported in elderly [30]. Together these data could, at least partially, explain the reduced effects of vaccination and immune responses against infections in elderly.

Chronically activated B cells express CD5 and CD43 $[31,32]$ and might trigger MBL onset. [33, 34]. MBL are found in healthy adult individuals, with an incidence that increases with age to roughly $10 \%$ of individuals $>65 y$ [35]. Based on their phenotypical association with MBL [20] and CLL [22], the increase of CD5 + CD43+ B cells upon aging might thus correlate with the higher risk of MBL and CLL clones in elderly. Another B cell subset related with chronic activation concerns $\mathrm{CD} 21^{\text {low }} \mathrm{B}$ cells, increased numbers of which can be found in patients showing chronic inflammation in the context of autoimmune disease [23]. As we excluded individuals with inflammatory and (auto)immune disease in our immunologically healthy cohort, unfortunately we could not link the higher number of $\mathrm{CD} 21^{\text {low }} \mathrm{B}$ cells in the $60-70 \mathrm{y}$ group to overt autoimmune disease occurrence. Nevertheless, increased numbers of $\mathrm{CD} 21^{\text {low }} \mathrm{B}$ cells in this age group might reflect an 
increased incidence of, yet undiagnosed, autoimmune diseases upon aging.

$B C R$ repertoire changes were most apparent in naive mature B cells of the $70+y$ group. Since naive mature B cells are not affected by exogenous antigen, this is most likely the result of changes in repertoire development and/ or output from the bone marrow. Whilst HCDR3 length, IGHD, and IGHJ usage remained stable in all three age categories, IGHV gene usage did reveal alterations. Interestingly, IGHV4-34 usage, a gene often associated with autoimmunity, was found to be reduced upon aging in these healthy individuals. Upon aging we also noticed a combined increased usage of IGHV5-51 and IGHV1-69, in line with a previous report [36]. IGHV1-69 has been associated with broadly neutralizing antibodies against amongst others influenza, HIV, hepatitis $\mathrm{C}$, and commensal bacteria antigens in the context of CLL [37].

Remarkably, IGHV gene usage in both the 50-70y and $70+y$ CLL patient groups most closely resembled IGHV gene usage in naive mature B cells of 50-70y, but not $70+$ $\mathrm{y}$, healthy individuals. One could speculate that CLL clones, even the ones in elderly CLL patients $(70+y)$, would have developed from B cells with a BCR repertoire of relatively younger age $(<70 \mathrm{y})$, but it might also reflect selection for IGHV specificities in the younger repertoire that could be predisposing for CLL development.

Stereotypic BCR, which are found in roughly one third of CLL clones, have previously been documented in RNA from a total lymphocyte pool of three healthy individuals (age 50, 69, and 69) [38, 39]. In this study we show that stereotypic BCR can be found in the normal $\mathrm{BCR}$ repertoire of both naive mature and antigen-experienced B cells. Interestingly, a frequently observed stereotypic BCR was the CLL\#2 BCR (IGHV3-21 in combination with IGHJ6 with a short HCDR3 length of 9 amino acids), which is the most common stereotypic BCR seen in CLL patients and is associated with an aggressive form of CLL $[17,40]$. Stereotypic CLL\#5 BCR (IGHV1-69, IGHD3-10/3-3, IGHJ6, 20 amino acids HCDR3) as well as stereotypic CLL\#64 BCR (IGHV3 subgroup genes, IGHD2 subgroup genes, IGHJ6, 21 amino acids HCDR3) [17] were relatively often detected in naive mature $B$ cells of especially individuals $70+y$. In antigen-experienced B cells, stereotypic CLL\#14 BCR (IGHV4-4, no specific IGHD, IGHJ4, and short 10 amino acids HCDR3) was frequently found. The possibility to detect stereotypic BCRs with short HCDR3 lengths in antigen-experienced B cell subpopulations would be in line with our observation that on average the complete memory BCR repertoire shows selection for shorter HCDR3 lengths in comparison with naive $B$ cells. Notably, other common CLL-associated stereotypic BCR, such as CLL\#4, CLL\#6, and CLL\#8 $\mathrm{BCR}$, could not be detected.
Even though the frequency of stereotypic $\mathrm{BCR}$ in healthy $\mathrm{B}$ cells shows a trend towards increase with age, stereotypic BCR can already be detected in cord blood (data not shown) and thus are to be considered as part of the normal $\mathrm{BCR}$ repertoire. Moreover, even though the increase of CLL-associated stereotypic BCR in the aging normal $\mathrm{BCR}$ repertoire might imply an increased predisposition for CLL development in elderly, it should be stressed that two thirds of CLL show heterogeneous BCR specificities that can also mediate derailment of B cells leading to CLL. Investigations into the presence of CLLassociated stereotypic BCR in healthy individuals should therefore be extended to larger datasets including more healthy individuals of all age groups, as well as to individuals suffering from chronic infection, immunodeficiency, or autoimmune disease. Such studies would allow to define the true impact of CLL-associated stereotypic BCR in CLL development.

\section{Conclusion}

We analyzed the peripheral B cell compartment and BCR repertoire during human ageing. Composition of the peripheral B cell compartment changes with ageing, with clear reductions in non-switched and $\mathrm{CD} 27+\mathrm{IgG}+$ switched memory B cells and plasma blasts in especially the $70+y$ group. The BCR repertoire is relatively stable until $70 \mathrm{y}$ age, whereafter differences in IGHV gene usage are seen. Upon ageing, an increase in the occurrence of particular CLL-associated stereotypic BCR is observed, potentially reflecting the occurrence of such BCR in CLL in elderly.

\section{Methods}

\section{Sample inclusion}

For B cell subpopulation analysis, peripheral blood (PB) of immunologically healthy individuals was obtained from pre-surgery patients (Dept. Orthopedics, Erasmus MC) with the following exclusion criteria: (auto)immune or inflammatory diseases; malignancies; usage of anti-inflammatory or immunosuppressive drugs; surgery in the past 30 days; alcohol and drug abuse. To increase the number of subjects per age group, additional samples from the SENEX healthy aging cohort (Rheumatology and Clinical Immunology, UMCG, Groningen, Netherlands), and samples from co-workers from the department were included. Subjects $(n=155)$ were divided into four age categories: < $50(n=47), 50-60(n=31), 60-70(n=45)$, and 70+ $(n=$ 32). For the BCR repertoire study, peripheral blood samples ( $n=5$ per age group) were additionally obtained from Sanquin Blood bank (Amsterdam, The Netherlands). Diagnostic samples from CLL patients were collected upon informed consent and anonymized for further usage. Written consent was obtained in accordance with the Declaration of Helsinki after medical ethics committee approval (MEC 2011-409, 2016-202, and 2,012,375). 
Immunophenotyping of $B$ cell subpopulations

Folllowing white blood cell count (WBC) measurement on a Coulter Ac.T diff analyzer (Beckman Coulter, Fullerton, CA, USA), flowcytometry was performed on whole blood [after red blood cell lysis with ammonium chloride] using an LSR Fortessa ${ }^{\text {Tm }}$ (BD Biosciences, San Jose, CA). Absolute cell counts of monocytes, natural killer (NK) cells, T cells, and B cells were calculated from WBC numbers using Infinicyt software (Cytognos, Salamanca, Spain). Lymphocytes were first gated based on FSC / SSC characteristics, and $\mathrm{B}$ cells were defined by expression of the pan-B cell marker CD19. Further gating was performed for defined CD19+ B cell subpopulations, i.e. transitional B cells (CD38hi/CD27-), naive mature B cells (CD38-/CD27-/IgM+/IgD+), non-switched memory B cells (CD38-/CD27+/IgM+/IgD+), IgM-only $B$ cells (CD38-/CD27+/IgM+/IgD-), switched memory B cells (CD38-/CD27+ or -/IgM-/IgD-IgG+ or IgA+ or IgE+), plasma blasts (CD38 $\left.{ }^{\mathrm{hi}} / \mathrm{CD} 27+\right), \mathrm{CD} 5+/ \mathrm{CD} 43+\mathrm{B}$ cells (CD38-/CD5+/CD43+), and CD21 ${ }^{\text {low }}$ B cells $\left(\mathrm{CD} 38^{\mathrm{dim}} /\right.$ CD21 ${ }^{\text {low }}$ ) according to published data [28] (see also (Additional file 6: Table S1), using 11-color flowcytometric stainings (Additional file 6: Table S3) and FACS DIVA software (BD Biosciences) for analysis.

\section{Sorting of $B$ cell subpopulations and DNA isolation}

PB mononuclear cells (PB-MNC) were isolated via Ficoll Paque gradient centrifugation. Subsequently, B cells were purified with human CD19 MicroBeads via AutoMACS (Myltenyi Biotech, Bergisch Gladbach, Germany). Next, several B cell subpopulations (naive mature, non-switched memory, IgM only, and CD27+/IgG+) were collected using a FACSAria cell sorter (BD Biosciences). Immediately after collection cells were lysed in RLT+ buffer (QIAGEN, Valencia, CA) complemented with $\beta$-mercapto-ethanol. Cells were used for DNA isolation with the DNA/RNA/ miRNA Easy kit (QIAGEN) and/or stored in $-80^{\circ} \mathrm{C}$ for later processing.

NGS-based BCR repertoire analysis of healthy individuals IGHV-IGHD-IGHJ rearrangements were amplified from 100 ng DNA of sorted B cell subpopulations (from $n=3-5$ healthy controls per age group; see Additional file 6: Table S3) using the BIOMED-2 IGH multiplex PCR with 6 IGHV primers and 1 IGHJ consensus primers that were extended with adapter sequences for NGS. PCR products were purified by gel extraction (QIAGEN) and subsequently by Agencourt AMpure XP beads (Beckman Coulter, Brea, CA). Concentrations were measured using Quant-iT Picogreen dsDNA assay (Invitrogen, Carlsbad, CA). PCR products were sequenced on a 454 GS junior (Roche, Branford, $\mathrm{CT}$ ), using the GS Junior Titanium emPCR, sequencing, and PicoTiterPlate kits (Roche), and partly on a MiSeq (Illumina, San Diego, CA) platform. Cross-validation experiments using B cells from healthy individuals showed comparability of data from both platforms (unpublished; see Additional file 7). Sequences were demultiplexed based on their multiplex identifier sequence and trimmed via the ImmunoGlobulin galaxy (IGGalaxy) pipeline [41]. FASTA files were uploaded in IMGT/High-V-QUEST (http://www. imgt.org/HighV-QUEST/login.action) and IMGT output files were further analyzed in the Antigen Receptor Galaxy pipeline, as described before [42].

For comparison purposes, a local Erasmus MC cohort of 920 CLL patients (mean 65y) was used, in which Sanger sequencing-based IGHV mutation status analysis was performed using the BIOMED-2 primers and protocol [43], and following ERIC interpretation guidelines [44].

IGHV-IGHJ circos plots were generated via the Circos Table Viewer (http://mkweb.bcgsc.ca/tableviewer/). CLLassociated stereotypic BCR in healthy control samples were defined using the ARResT/AssignSubsets tool (http://tools. bat.infspire.org/arrest/assignsubsets/).

\section{Statistical analysis}

Significant differences in relative and absolute numbers of lymphocyte subpopulations and IGH SHM levels between age groups were determined using the MannWhitney U test. Differences in HCDR3 lengths were evaluated using Kolmogorov-Smirnov statistics. Relative IGHV gene usage and relative frequencies of stereotyped $B C R$ between different age groups were analyzed using a two-way ANOVA with multiple comparisons. Overall IGHV gene usage between B cell subpopulations and CLL clones was analyzed using the Fisher's exact test. A $p$-value $<0.05$ was considered significant. Statistics were performed in GraphPad Prism v5.0 (La Jolla, CA).

\section{Additional files}

Additional file 1: Figure S1. Frequencies and absolute numbers of $T$ cell subsets and NK cells to validate the cohort for evaluating peripheral blood B cell subpopulations upon aging. (PDF $472 \mathrm{~kb}$ )

Additional file 2: Figure S2. Scheme of different human B-cell subpopulations in peripheral blood. (PDF $209 \mathrm{~kb}$ )

Additional file 3: Figure S3. Relative frequencies of B cell subpopulations in peripheral blood upon aging. (PDF $450 \mathrm{~kb}$ )

Additional file 4: Figure S4. No difference in HCDR3 lengths of antigen-experienced B cells upon aging. (PDF 239 kb)

Additional file 5: Figure S5. Minor differences in BCR repertoire of antigenexperienced B cell subpopulations in different age groups. (PDF 8560 kb)

Additional file 6: Table S1. Definition of human B cell subpopulations. Table S2 Overview of productive and unique IGH sequences in NGS analysis. Table S3 Composition of different antibody panels for staining B cell subpopulations. (DOCX $26 \mathrm{~kb}$ )

Additional file 7: Cross-validation of IGH assay between different NGS platforms. (DOCX $32 \mathrm{~kb}$ )

\section{Abbreviations}

BCR: B cell receptor; CLL: Chronic lymphocytic leukemia; IG: Immunoglobulin; IGH: Immunoglobulin heavy chain gene complex; IGHD: Immunoglobulin 
heavy chain diversity gene; IGHJ: Immunoglobulin heavy chain joining gene; IGHV: Immunoglobulin heavy chain variable gene; MBL: Monoclonal B cell lymphocytosis; SHM: Somatic hypermutation

\section{Acknowledgements}

The authors wish to thank Michèle van der Klift, Irene van der Linden, Kim Heezen, Tamara Wabeke, Joyce Schilperoord-Vermeulen, Ellen van GastelMol, Diana van den Heuvel, and Pauline van Schouwenburg for excellent technical and analytical support. The research for this manuscript was performed within the framework of the Erasmus MC Postgraduate School Molecular Medicine.

\section{Authors' contributions}

AFM and AWL drafted the study; AFM, MdJ, ILMWT, HIJ, MvdB, and WvIJ performed experiments and obtained data; AFM, JANV, WHA, EB, and AMHB were responsible for selecting cases and defining data; AFM, CT, ND, RS, RWH, JJMVD, and AWL analyzed and interpreted data; AFM, RWH, JJMVD, and AWL wrote the manuscript. All authors read and approved the final manuscript.

\section{Funding}

This study was financially supported through an unrestricted grant from Roche-Genentech (to AWL and JJMvD), and a Ministry of Health of the Czech Republic grant 16-34272A (to ND).

\section{Availability of data and materials}

The datasets used and/or analysed during the current study are available from the corresponding author on reasonable request.

\section{Ethics approval and consent to participate}

Ethical approval and written consent to participate was obtained from the medical ethics committee of Erasmus MC, Rotterdam (MEC 2011-409, 2016202) and UMCG, Groningen (2012375).

\section{Consent for publication}

Written consent for publication was obtained from the medical ethics committee of Erasmus MC, Rotterdam (MEC 2011-409, 2016-202) and UMCG, Groningen (2012375).

\section{Competing interests}

The authors declare that they have no competing interests.

\section{Author details}

'Department Immunology, Laboratory Medical Immunology, Erasmus MC, Dr. Molewaterplein 40, 3015, GD, Rotterdam, The Netherlands. 'Present Address: Department Immunohematology and Blood Transfusion, Leiden University Medical Center, Leiden, The Netherlands. ${ }^{3}$ Central European Institute of Technology, Masaryk University, Brno, Czech Republic. ${ }^{4}$ Department Internal Medicine, University Schleswig-Holstein, Kiel, Germany. ${ }^{5}$ Department Pulmonary Medicine, Erasmus MC, Rotterdam, The Netherlands. ${ }^{6}$ Present Address: Department Pediatrics, Leiden University Medical Center, Leiden, The Netherlands. 'Biomics Core Facility, Erasmus MC, Rotterdam, The Netherlands. ${ }^{8}$ Department Orthopedics, Erasmus MC, Rotterdam, The Netherlands. ${ }^{9}$ Department Rheumatology and Clinical Immunology, University Medical Center Groningen, Groningen, The Netherlands.

Received: 28 February 2019 Accepted: 21 August 2019

Published online: 28 August 2019

\section{References}

1. Grubeck-Loebenstein B, Della Bella S, lorio AM, Michel JP, Pawelec G, Solana R. Immunosenescence and vaccine failure in the elderly. Aging Clin Exp Res. 2009;21(3):201-9.

2. Ademokun A, Wu YC, Dunn-Walters D. The ageing B cell population: composition and function. Biogerontology. 2010;11(2):125-37.

3. Goronzy JJ, Weyand CM. Understanding immunosenescence to improve responses to vaccines. Nat Immunol. 2013;14(5):428-36.

4. Johnson SA, Rozzo SJ, Cambier JC. Aging-dependent exclusion of antigeninexperienced cells from the peripheral B cell repertoire. J Immunol. 2002: 168(10):5014-23.
5. Williams GT, Jolly CJ, Kohler J, Neuberger MS. The contribution of somatic hypermutation to the diversity of serum immunoglobulin: dramatic increase with age. Immunity. 2000;13(3):409-17.

6. Chong Y, Ikematsu H, Yamaji K, Nishimura M, Nabeshima S, Kashiwagi S, et al. CD27(+) (memory) B cell decrease and apoptosis-resistant CD27(-) (naive) B cell increase in aged humans: implications for age-related peripheral B cell developmental disturbances. Int Immunol. 2005;17(4):383-90.

7. Frasca D, Landin AM, Lechner SC, Ryan JG, Schwartz R, Riley RL, et al. Aging down-regulates the transcription factor E2A, activation-induced cytidine deaminase, and Ig class switch in human B cells. J Immunol. 2008;180(8):5283-90,

8. Shi Y, Yamazaki T, Okubo Y, Uehara Y, Sugane K, Agematsu K. Regulation of aged humoral immune defense against pneumococcal bacteria by $\operatorname{lgM}$ memory B cell. J Immunol. 2005;175(5):3262-7.

9. Veneri D, Ortolani R, Franchini M, Tridente G, Pizzolo G, Vella A. Expression of CD27 and CD23 on peripheral blood B lymphocytes in humans of different ages. Blood Transfus. 2009;7(1):29-34.

10. Morbach H, Eichhorn EM, Liese JG, Girschick HJ. Reference values for B cell subpopulations from infancy to adulthood. Clin Exp Immunol. 2010;162(2):271-9.

11. Colonna-Romano G, Aquino A, Bulati M, Di Lorenzo G, Listi F, Vitello S, et al. Memory B cell subpopulations in the aged. Rejuvenation Res. 2006;9(1):149-52.

12. van Gent $R$, van Tilburg CM, Nibbelke EE, Otto SA, Gaiser JF, JanssensKorpela PL, et al. Refined characterization and reference values of the pediatric T- and B-cell compartments. Clin Immunol. 2009;133(1):95-107.

13. Gibson KL, Wu YC, Barnett Y, Duggan O, Vaughan R, Kondeatis E, et al. B-cell diversity decreases in old age and is correlated with poor health status. Aging Cell. 2009;8(1):18-25.

14. Cancro MP, Hao Y, Scholz JL, Riley RL, Frasca D, Dunn-Walters DK, et al. B cells and aging: molecules and mechanisms. Trends Immunol. 2009;30(7): 313-8.

15. Chong Y, Ikematsu H, Yamaji K, Nishimura M, Kashiwagi S, Hayashi J. Agerelated accumulation of $\lg V(H)$ gene somatic mutations in peripheral $B$ cells from aged humans. Clin Exp Immunol. 2003;133(1):59-66.

16. Kolar GR, Mehta D, Wilson PC, Capra JD. Diversity of the Ig repertoire is maintained with age in spite of reduced germinal Centre cells in human tonsil lymphoid tissue. Scand J Immunol. 2006;64(3):314-24.

17. Agathangelidis A, Darzentas N, Hadzidimitriou A, Brochet X, Murray F, Yan $\mathrm{XJ}$, et al. Stereotyped B-cell receptors in one-third of chronic lymphocytic leukemia: a molecular classification with implications for targeted therapies. Blood. 2012;119(19):4467-75.

18. Forconi F, Potter KN, Wheatley I, Darzentas N, Sozzi E, Stamatopoulos K, et al. The normal IGHV1-69-derived B-cell repertoire contains stereotypic patterns characteristic of unmutated CLL. Blood. 2010;115(1):71-7.

19. Lin Y, Kim J, Metter EJ, Nguyen H, Truong T, Lustig A, et al. Changes in blood lymphocyte numbers with age in vivo and their association with the levels of cytokines/cytokine receptors. Immun Ageing. 2016;13:24.

20. Nieto WG, Teodosio C, Lopez A, Rodriguez-Caballero A, Romero A, Barcena $P$, et al. Non-CLL-like monoclonal B-cell lymphocytosis in the general population: prevalence and phenotypic/genetic characteristics. Cytometry B Clin Cytom. 2010;78(Suppl 1):S24-34.

21. Griffin DO, Holodick NE, Rothstein TL. Human B1 cells in umbilical cord and adult peripheral blood express the novel phenotype CD20+ CD27+ CD43+ CD70. J Exp Med. 2011;208(1):67-80.

22. Seifert M, Sellmann L, Bloehdorn J, Wein F, Stilgenbauer S, Durig J, et al. Cellular origin and pathophysiology of chronic lymphocytic leukemia. J Exp Med. 2012;209(12):2183-98.

23. Thorarinsdottir K, Camponeschi A, Gjertsson I, Martensson IL. CD21 -/low B cells: a snapshot of a unique B cell subset in health and disease. Scand J Immunol. 2015;82(3):254-61.

24. Bystry $V$, Agathangelidis $A$, Bikos $V$, Sutton $L A$, Baliakas $P$, Hadzidimitriou $A$, et al. ARResT/AssignSubsets: a novel application for robust subclassification of chronic lymphocytic leukemia based on B cell receptor IG stereotypy. Bioinformatics. 2015;31(23):3844-6.

25. Duggal NA, Upton J, Phillips AC, Sapey E, Lord JM. An age-related numerical and functional deficit in CD19(+) CD24(hi) CD38(hi) B cells is associated with an increase in systemic autoimmunity. Aging Cell. 2013;12(5):873-81.

26. van der Geest KS, Lorencetti PG, Abdulahad WH, Horst G, Huitema M, Roozendaal C, et al. Aging-dependent decline of IL-10 producing B cells coincides with production of antinuclear antibodies but not rheumatoid factors. Exp Gerontol. 2016;75:24-9.

27. Kruetzmann S, Rosado MM, Weber $\mathrm{H}$, Germing U, Tournilhac O, Peter $\mathrm{HH}$, et al. Human immunoglobulin M memory B cells controlling Streptococcus 
pneumoniae infections are generated in the spleen. J Exp Med. 2003;197(7): 939-45.

28. Berkowska MA, Driessen GJ, Bikos V, Grosserichter-Wagener C, Stamatopoulos K, Cerutti A, et al. Human memory B cells originate from three distinct germinal center-dependent and -independent maturation pathways. Blood. 2011;118(8):2150-8.

29. Caraux A, Klein B, Paiva B, Bret C, Schmitz A, Fuhler GM, et al. Circulating human $B$ and plasma cells. Age-associated changes in counts and detailed characterization of circulating normal CD138- and CD138+ plasma cells. Haematologica. 2010;95(6):1016-20.

30. Lock RJ, Unsworth DJ. Immunoglobulins and immunoglobulin subclasses in the elderly. Ann Clin Biochem. 2003;40(Pt 2):143-8.

31. Mageed RA, Garaud S, Taher TE, Parikh K, Pers JO, Jamin C, et al. CD5 expression promotes multiple intracellular signaling pathways in B lymphocyte. Autoimmun Rev. 2012;11(11):795-8.

32. Gary-Gouy H, Harriague J, Bismuth G, Platzer C, Schmitt C, Dalloul AH. Human CD5 promotes B-cell survival through stimulation of autocrine IL-10 production. Blood. 2002;100(13):4537-43.

33. Perez-Andres M, Grosserichter-Wagener C, Teodosio C, van Dongen JJ, Orfao A, van Zelm MC. The nature of circulating CD27+CD43+ B cells. J Exp Med. 2011;208(13):2565-6.

34. Covens K, Verbinnen B, Geukens N, Meyts I, Schuit F, Van Lommel L, et al. Characterization of proposed human B-1 cells reveals pre-plasmablast phenotype. Blood. 2013;121(26):5176-83.

35. Shim YK, Rachel JM, Ghia P, Boren J, Abbasi F, Dagklis A, et al. Monoclonal B-cell lymphocytosis in healthy blood donors: an unexpectedly common finding. Blood. 2014;123(9):1319-26.

36. Martin V, Wu YC, Kipling D, Dunn-Walters DK. Age-related aspects of human $\lg M(+)$ B cell heterogeneity. Ann N Y Acad Sci. 2015;1362:153-63.

37. Hwang KK, Trama AM, Kozink DM, Chen X, Wiehe K, Cooper AJ, et al. IGHV169 B cell chronic lymphocytic leukemia antibodies cross-react with HIV-1 and hepatitis $C$ virus antigens as well as intestinal commensal bacteria. PLoS One. 2014:9(3):e90725.

38. Henriques A, Rodriguez-Caballero A, Nieto WG, Langerak AW, Criado I, Lecrevisse $\mathrm{Q}$, et al. Combined patterns of IGHV repertoire and cytogenetic/ molecular alterations in monoclonal B lymphocytosis versus chronic lymphocytic leukemia. PLoS One. 2013;8(7):e67751.

39. DeSantis CE, Lin CC, Mariotto AB, Siegel RL, Stein KD, Kramer JL, et al. Cancer treatment and survivorship statistics, 2014. CA Cancer J Clin. 2014: 64(4):252-71.

40. Baliakas P, Agathangelidis A, Hadzidimitriou A, Sutton LA, Minga E, Tsanousa A, et al. Not all IGHV3-21 chronic lymphocytic leukemias are equal: prognostic considerations. Blood. 2015;125(5):856-9.

41. Moorhouse MJ, van Zessen D, IJ H, Hiltemann S, Horsman S, van der Spek PJ, et al. ImmunoGlobulin galaxy (IGGalaxy) for simple determination and quantitation of immunoglobulin heavy chain rearrangements from NGS BMC Immunol. 2014;15:59.

42. IJspeert H, van Schouwenburg PA, van Zessen D, Pico-Knijnenburg I, Stubbs $A P$, van der Burg M. Antigen receptor galaxy: a user-friendly, web-based tool for analysis and visualization of $\mathrm{T}$ and $\mathrm{B}$ cell receptor repertoire data. Immunol. 2017;198(10):4156-65.

43. van Dongen JJ, Langerak AW, Bruggemann M, Evans PA, Hummel M, Lavender FL, et al. Design and standardization of PCR primers and protocols for detection of clonal immunoglobulin and T-cell receptor gene recombinations in suspect lymphoproliferations: report of the BIOMED-2 concerted action BMH4-CT98-3936. Leukemia. 2003;17(12):2257-317.

44. Langerak AW, Davi F, Ghia P, Hadzidimitriou A, Murray F, Potter KN, et al. Immunoglobulin sequence analysis and prognostication in CLL: guidelines from the ERIC review board for reliable interpretation of problematic cases. Leukemia. 2011;25(6):979-84.

\section{Publisher's Note}

Springer Nature remains neutral with regard to jurisdictional claims in published maps and institutional affiliations.

Ready to submit your research? Choose BMC and benefit from:

- fast, convenient online submission

- thorough peer review by experienced researchers in your field

- rapid publication on acceptance

- support for research data, including large and complex data types

- gold Open Access which fosters wider collaboration and increased citations

- maximum visibility for your research: over $100 \mathrm{M}$ website views per year

At BMC, research is always in progress.

Learn more biomedcentral.com/submissions 